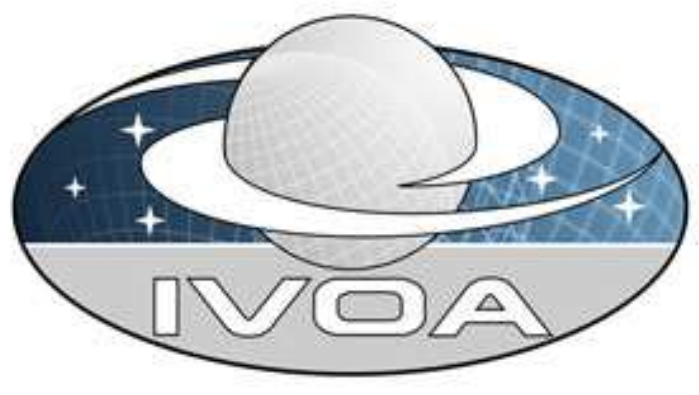

International

Virtual

Observatory

Alliance

\title{
The UCD1+ controlled vocabulary Version 1.23
}

\section{IVOA Recommendation 2007 April 2}

\section{This version:}

http://www.ivoa.net/Documents/REC/UCD/UCDlist-20070402.html

\section{Latest version:}

http://www.ivoa.net/Documents/latest/UCDlist.html

\section{Previous version(s):}

Editor(s):

http://www.ivoa.net/Documents/REC/UCD/UCDlist-20051231.html

A. Preite Martinez, S. Derriere

\section{Author(s):}

Andrea Preite Martinez (andrea.preitemartinez@iasf-roma.inaf.it),

Sebastien Derriere (derriere@astro.u-strasbg.fr),

Nausicaa Delmotte (ndelmot@eso.org),

Norman Gray (norman@astro.gla.ac.uk),

Robert Mann (rgm@roe.ac.uk),

Jonathan McDowell (jcm@cfa.harvard.edu),

Thomas Mc Glynn (Thomas.A.McGlynn@nasa.gov),

François Ochsenbein (francois@astro.u-strasbg.fr),

Pedro Osuna (Pedro.Osuna@esa.int),

Guy Rixon (gtr@ast.cam.ac.uk),

Roy Williams (roy@cacr.caltech.edu) 


\begin{abstract}
This document describes the list of controlled terms used to build the Unified Content Descriptors, Version 1+ (UCD1+). The document describing the UCD1+ can be found at the URL: http://www.ivoa.net/Documents/latest/UCD.html. This document reviews the structure of the UCD1+ and presents the current vocabulary.
\end{abstract}

\title{
Status of This Document
}

This is an IVOA Recommendation. This document has been produced by the IVOA Semantics Working Group. It has been reviewed by IVOA Members and other interested parties, and has been endorsed by the IVOA Executive Committee as an IVOA Recommendation. It is a stable document and may be used as reference material or cited as a normative reference from another document. IVOA's role in making the Recommendation is to draw attention to the specification and to promote its widespread deployment. This enhances the functionality and interoperability inside the Astronomical Community.

A list of current IVOA Recommendations and other technical documents can be found at http://www.ivoa.net/Documents/.

\section{Acknowledgements}

This document is based on the W3C documentation standards as adapted for the IVOA.

\section{Contents}

The UCD1+ controlled vocabulary Version 1.23

1 Definition of atoms and words 3

1.1 Definition of atoms 3

1.2 Definition of words 3

2 The structure of the UCD1+ tree 4

Appendix A: List of valid words $\quad 5$

Appendix B: Changes from previous versions $\quad 15$

Changes from v1.22 15

Changes from v1.21 15

Changes from v1.2 15

Changes from v1.11 (Rec20051231)

Changes from v1.10 16

Changes from v1.02 17

$\begin{array}{lr}\text { Changes from v1.01 } & 17\end{array}$

Changes from v1.00 17

Changes from v0.2 19

Changes from v0.1 20

$\begin{array}{ll}\text { References } & 21\end{array}$ 


\section{Definition of atoms and words}

A UCD is a string which contains textual tokens called 'words', separated by semicolons(;). A word is composed of 'atoms', separated by periods(.). The hierarchy is as follows:

$$
\text { atoms --> words --> composed words }
$$

UCD1+ are either single words, or a composition of several words.

UCDs are "controlled" (through a process that is also indicated in the reference document above). Control is exercised at the level of words (UCD1+) and at the level of the vocabulary (atoms) used to form words. A consistent list of atoms will be mantained, making sure that the same atom always means the same thing, even if used in combination with different other atoms.

\subsection{Definition of atoms}

Atoms are defined following these guidelines:

1. Abbreviations are kept to a minimum, and only if the result is not ambiguous. (ra, dec are acceptable, but $t$ is ambiguous: time and temperature are used instead.)

2. Atoms are not hyphenated. The separation is marked by a capital letter to help readability (position angle $=$ posAng) unless the composed word has a well known acronym (signal to noise ratio $=$ snr) or short form (standard deviation $=$ stdev). There are only two exception to this rule: (i) the X-ray band (em.X-ray) and (ii) the frequency / wavelength intervals defining regions of the e.m. spectrum (e.g. em.radio.3-6GHz).

\subsection{Definition of words}

The list of UCD1+ words presented in this document was initially generated applying the rules and recommendations of PR-UCD-20040823 to catalogues/tables in VizieR. The original motivation was to transform old UCD1 into an improved version, trying to build a list of combinations of new words that could describe all the existing UCD1 terms.

The list of UCD1+ words is maintained by the UCD Scientific Board, following the procedure defined in the UCD Recommendation document (http://www.ivoa.net/Documents/latest/UCD.html), and described in detail in http://www.ivoa.net/Documents/latest/UCDlistMaintenance.html . 


\section{The structure of the UCD1+ tree}

All existing UCD1+ words are grouped into 12 main categories. These categories are expressed by the first atom of the word, whose possible values are:

1. arith (arithmetics)

This section includes concepts involving or indicating some mathematical operation performed on the primary 'concept' or just the presence of an arithmetic factor or operator.

2. em (electromagnetic spectrum)

This section describes the electromagnetic spectrum, either in a monochromatic way or in predefined intervals. The complete list of proposed bands (in seven classical regions of the e.m. spectrum: radio, millimeter, infrared, optical, ultraviolet, $x$-ray and gamma-ray), can be found in the document Note-EMSpectrum-20040520

3. instr (instrument)

This section includes all quantities related to astronomical instrumentation, e.g. detectors (plates, CCDs, etc.), spectrographs, and telescopes (including observatories or missions), etc.

4. meta (metadata)

This section includes all the information that is not coming directly from a measurement, and information that could not be included in other sections.

5. obs (observation)

In principle under this section should go all words describing an observation (the name of the observer or PI, the observing conditions, the name of the field). In practice, the section is very 'thin' and could be deleted, if the sparse content could be housed elsewere.

6. phot (photometry)

All the words describing photometric measures are included in this section. The definitions distinguish between a flux density (flux per unit frequency interval), a flux density integrated over a given e.m. interval (flux if expressed linearly, mag if expressed by a log), or a flux expressed in counts/s (if the setup of the detector is photon counting observing mode). 'Colors', which are differences of magnitudes (i.e. ratios of fluxes) measured in different bandpasses, are also included. 
7. phys (physics)

This section includes atomic and molecular data (mainly used for spectroscopy) and basic physical quantities (temperature, mass, gravity, luminosity, etc.)

8. pos (positional data)

This section describes all quantities related to the position of an object on the sky:

- Angular coordinates, and projections from spherical to rectangular systems.

- Angular measurements in general (the angular size of an object is in this section, its linear size is in the phys section).

- The WCS FITS keywords.

9. spect (spectral data)

For historical reasons, photometric data taken in narrow spectral bands with instruments called spectrographs are classified as spectroscopic data. These definitions should not be confused with those in the em category. represents the independent variable, or dispersion axis, and phot and spect describe the dependent variable, or flux axis.

10. sirc (source)

This is a rather generic section, mainly devoted to source classifications. Variability, orbital, and velocity data are also included in this section.

11. stat (statistics)

This section includes statistical information on measurements.

12. time (time)

Quantities related to time (age, date, period, etc.) are described in this section.

\section{Appendix A: List of valid words}

All words are preceded by a 'syntax' code that can help in the process of building composed UCD1+. 
1. The code "P" means that the word can only be used as "primary" or first word;

2. "S" stands for only secondary: the word cannot be used as the first word to describe a single quantity;

3. "Q" means that the word can be used indifferently as first or secondary word;

4. "E" means a photometric quantity, and can be followed by a word describing a part of the electromagnetic spectrum

5. " $\mathrm{C}$ " is a colour index, and can be followed by two successive word describing a part of the electromagnetic spectrum;

6. "V" stands for vector. Such a word can be followed by another describing the axis or reference frame in which the measurement is done

\begin{tabular}{|c|c|}
\hline & $\begin{array}{l}\text { | arith } \\
\text { | arith.diff }\end{array}$ \\
\hline$P$ & arith.factor \\
\hline$P$ & arith.grad \\
\hline$P \mid$ & arith.rate \\
\hline $\mathrm{S}$ & arith.ratio \\
\hline Q & | arith.zp \\
\hline $\mathrm{S}$ & em \\
\hline $\mathrm{S}$ & | em.radio \\
\hline S & | em.radio.20-100MHz \\
\hline $\mathrm{S} \mid$ & | em.radio.100-200MHz \\
\hline S I & | em.radio. $200-400 \mathrm{MHz}$ \\
\hline S & | em.radio.400-750MHz \\
\hline $\mathrm{S} \mid$ & | em.radio.750-1500MHz \\
\hline $\mathrm{S}$ & | em.radio. $1500-3000 \mathrm{MHz}$ \\
\hline S I & | em.radio.3-6GHz \\
\hline S & | em.radio.6-12GHz \\
\hline $\mathrm{S} \mid$ & | em.radio.12-30GHz \\
\hline S I & em.mm \\
\hline S & | em.mm.30-50GHz \\
\hline $\mathrm{S} \mid$ & | em.mm.50-100GHz \\
\hline $\mathrm{S}$ & | em.mm.100-200GHz \\
\hline $\mathrm{S}$ & em.mm.200-400GHz \\
\hline $\mathrm{S}$ & | em.mm.400-750GHz \\
\hline S & em.mm.750-1500GHz \\
\hline S I & em.mm.1500-3000GHz \\
\hline $\mathrm{S}$ & em.IR \\
\hline S I & | em.IR.J \\
\hline $\mathrm{S} \mid$ & em.IR.H \\
\hline $\mathrm{S}$ & em.IR.K \\
\hline S & | em.IR.3-4um \\
\hline S & em.IR.4-8um \\
\hline S I & | em.IR.8-15um \\
\hline $\mathrm{S}$ & em.IR.15-30um \\
\hline S I & | em.IR.30-60um \\
\hline S I & em.IR.60-100um \\
\hline $\mathrm{S}$ & | em.IR.NIR \\
\hline S & | em.IR.MIR \\
\hline S & | em.IR.FIR \\
\hline$S$ & | em.opt \\
\hline S 1 & | em.opt.U \\
\hline
\end{tabular}

| Arithmetic quantities

| Difference between two quantities described by the same UCD

Numerical factor

| Gradient

| Rate (per time unit)

Ratio between two quantities described by the same UCD

| Zero point

| Electromagnetic spectrum

Radio part of the spectrum

Radio between 20 and $100 \mathrm{MHz}$

| Radio between 100 and $200 \mathrm{MHz}$

| Radio between 200 and $400 \mathrm{MHz}$

Radio between 400 and $750 \mathrm{MHz}$

| Radio between 750 and $1500 \mathrm{MHz}$

Radio between 1500 and $3000 \mathrm{MHz}$

| Radio between 3 and $6 \mathrm{GHz}$

| Radio between 6 and $12 \mathrm{GHz}$

| Radio between 12 and $30 \mathrm{GHz}$

| Millimetric part of the spectrum

Millimetric between 30 and $50 \mathrm{GHz}$

Millimetric between 50 and $100 \mathrm{GHz}$

| Millimetric between 100 and $200 \mathrm{GHz}$

Millimetric between 200 and $400 \mathrm{GHz}$

| Millimetric between 400 and $750 \mathrm{GHz}$

| Millimetric between 750 and $1500 \mathrm{GHz}$

Millimetric between 1500 and $3000 \mathrm{GHz}$

I Infrared part of the spectrum

I Infrared between 1.0 and 1.5 micron

Infrared between 1.5 and 2 micron

Infrared between 2 and 3 micron

Infrared between 3 and 4 micron

Infrared between 4 and 8 micron

Infrared between 8 and 15 micron

| Infrared between 15 and 30 micron

| Infrared between 30 and 60 micron

Infrared between 60 and 100 micron

| Near-Infrared, 1-5 microns

| Medium-Infrared, 5-30 microns

| Far-Infrared, 30-1000 microns

Optical part of the spectrum

| Optical band between 300 and 400 nm 
S | em.opt.B

S | em.opt.V

$S$ | em.opt.R

$\mathrm{S}$ | em.opt.I

S | em.UV

S | em.UV.10-50nm

$\mathrm{S}$ | em.UV.50-100nm

$\mathrm{S}$ | em.UV.100-200nm

S | em.UV.200-300nm

S l em.UV.FUV

$S$ | em.X-ray

S | em.X-ray.soft

S | em.X-ray.medium

S | em.X-ray.hard

S | em.gamma

$\mathrm{S}$ | em.gamma.soft

$\mathrm{S}$ | em.gamma.hard

$\mathrm{S}$ | em.line

$\mathrm{S}$ | em.line.Brgamma

$\mathrm{S}$ | em.line. HI

$\mathrm{S}$ | em.line.Halpha

S | em.line.Hbeta

$\mathrm{S}$ | em.line.Hgamma

$\mathrm{S}$ | em.line.Hdelta

S | em.line.Lyalpha

$\mathrm{S}$ | em.line.OIII

$\mathrm{S} \mid$ em.line.CO

$\mathrm{Q} \mid$ em.bin

$Q$ | em.energy

$Q$ | em.freq

$Q$ | em.wavenumber

$\mathrm{Q}$ | em.wl

$\mathrm{Q}$ | em.wl.central

$\mathrm{Q}$ | em.wl.effective

$\mathrm{Q}$ | instr

E | instr.background

Q | instr.bandpass

Q | instr.bandwidth

Q | instr.baseline

$S$ | instr.beam

$Q$ | instr.calib

$S$ | instr.det

$\mathrm{Q}$ | instr.det.noise

Q | instr.det.psf

$\mathrm{Q}$ | instr.det.qe

$\mathrm{Q}$ | instr.dispersion

$S$ | instr.filter

$S$ | instr.fov

S | instr.obsty

Q | instr.obsty.seeing

$Q$ | instr.offset

$\mathrm{Q}$ | instr.order

$\mathrm{Q}$ | instr.param

$S$ | instr.pixel

$\mathrm{S}$ | instr.plate

$\mathrm{Q}$ | instr.plate.emulsion
I Optical band between 400 and 500 nm

| Optical band between 500 and $600 \mathrm{~nm}$

| Optical band between 600 and 750 nm

| Optical band between 750 and 1000 nm

| Ultraviolet part of the spectrum

| Ultraviolet between 10 and $50 \mathrm{~nm}$

| Ultraviolet between 50 and $100 \mathrm{~nm}$

| Ultraviolet between 100 and $200 \mathrm{~nm}$

| Ultraviolet between 200 and 300 nm

Far-Ultraviolet

X-ray part of the spectrum

Soft X-ray (0.12 - $2 \mathrm{keV})$

Medium X-ray (2 - $12 \mathrm{keV})$

Hard X-ray (12 - $120 \mathrm{keV})$

Gamma rays part of the spectrum

Soft gamma ray (120 - $500 \mathrm{keV})$

| Hard gamma ray (>500 keV)

| Designation of major atomic lines

| Bracket gamma line

| $21 \mathrm{~cm}$ hydrogen line

| $\mathrm{H}$-alpha line

H-beta line

H-gamma line

H-delta line

| Hydrogen Lyman alpha line

[ [OIII] line whose rest wl is $500.7 \mathrm{~nm}$

CO radio line, e.g. $12 \mathrm{CO}(1-0)$ at $115 \mathrm{GHz}$

| Channel / instrumental spectral bin coordinate (bin number)

| Energy value in the em frame

Frequency value in the em frame

| Wavenumber value in the em frame

I Wavelength value in the em frame

Central wavelength

| Effective wavelength

| Instrument

Instrumental background

Bandpass (e.g.: band name) of instrument

| Bandwidth of the instrument

| Baseline for interferometry

Beam

| Calibration parameter

| Detector

Instrument noise

| Point Spread Function

| Quantum efficiency

| Dispersion of a spectrograph

| Filter

| Field of view

| Observatory, satellite, mission

Seeing

Offset angle respect to main direction of observation

| Spectral order in a spectrograph

Various instrumental parameters

| Pixel (default size: angular)

Photographic plate

| Plate emulsion 


\begin{tabular}{|c|c|c|}
\hline & | instr.precision & Instrument precision \\
\hline$\tilde{Q}$ & instr.saturation & Instrument saturation threshold \\
\hline Q & instr.scale & Instrument scale (for CCD, plate, image) \\
\hline Q & instr.sensitivity & Instrument sensitivity, detection threshold \\
\hline$Q$ & instr.setup & Instrument configuration or setup \\
\hline Q & instr.skyLevel & Sky level \\
\hline$\vec{Q}$ & instr.skyTemp & Sky temperature \\
\hline Q & instr.tel & Telescope \\
\hline$\hat{Q}$ & instr.tel.focalLength & Telescope focal length \\
\hline$P \mid$ & meta & Metadata \\
\hline$P \mid$ & meta.abstract & Abstract (of paper, proposal,etc.) \\
\hline$P$ & meta.bib & Bibliographic reference \\
\hline$P \mid$ & meta.bib.author & Author name \\
\hline$P$ & meta.bib.bibcode & Bibcode \\
\hline$P$ & meta.bib.fig & Figure in a paper \\
\hline $\mathrm{P}$ & meta.bib.journal & Journal name \\
\hline$P$ & meta.bib.page & Page number \\
\hline$P \mid$ & meta.bib.volume & Volume number \\
\hline$P$ & meta.code & Code or flag \\
\hline$P$ & meta.code.class & Classification code \\
\hline$P$ & meta.code.error & limit uncertainty error flag \\
\hline $\mathrm{P}$ & meta.code.member & Membership code \\
\hline $\mathrm{P}$ & meta.code.mime & MIME type \\
\hline$P$ & meta.code.multip & Multiplicity or binarity flag \\
\hline$P$ & meta.code.qual & Quality, precision, reliability flag or code \\
\hline$P$ & meta.code.status & Status code (e.g.: status of a proposal/observation) \\
\hline $\mathrm{P}$ & meta.cryptic & Unknown or impossible to understand quantity \\
\hline $\mathrm{P}$ & meta.curation & Identity of man/organization responsible for the data \\
\hline Q & meta.dataset & Dataset, archive \\
\hline Q & meta.email & Curation/contact e-mail \\
\hline S & meta.file & File \\
\hline S & meta.fits & FITS standard \\
\hline $\mathrm{P}$ & meta.id & Identifier, name or designation \\
\hline$P$ & meta.id.assoc & Identifier of associated counterpart \\
\hline $\mathrm{P}$ & meta.id.Col & Name of Co-Investigator \\
\hline $\mathrm{P}$ & meta.id.cross & Cross identification \\
\hline $\mathrm{P}$ & meta.id.parent & Identification of parent source \\
\hline$P$ & meta.id.part & Part of identifier, suffix or sub-component \\
\hline$P$ & meta.id.PI & Name of Principal Investigator \\
\hline S & meta.main & Main value of something \\
\hline S & meta.modelled & Quantity was produced by a model \\
\hline$P$ & meta.note & Note or remark (longer than a code or flag) \\
\hline $\mathrm{P}$ & meta.number & Number (of things; e.g. nb of object in an image) \\
\hline $\mathrm{P}$ & meta.record & Record number \\
\hline$P$ & meta.ref & Reference, or origin \\
\hline Q & meta.ref.ivorn & IVORN, Int. VO Resource Name (ivo://) \\
\hline$\tilde{Q}$ & meta.ref.uri & URI, universal resource identifier \\
\hline$P$ & meta.ref.url & URL, web address, service endpoint \\
\hline S & meta.software & Software used in generating data \\
\hline S & meta.table & Table or catalogue \\
\hline $\mathrm{P}$ & meta.title & Title or explanation \\
\hline Q & meta.ucd & UCD \\
\hline$P$ & meta.unit & Unit \\
\hline $\mathrm{P}$ & meta.version & Version \\
\hline $\mathrm{S}$ & obs & Observation \\
\hline Q & obs.airMass & Airmass \\
\hline
\end{tabular}


S | obs.atmos

Q | obs.atmos.extinction

Q | obs.atmos. refractAngle

$S$ | obs.calib

S | obs.calib.flat

$S$ obs.exposure

$\mathrm{S}$ | obs.field

S | obs.image

Q | obs.observer

Q | obs.param

S | obs.proposal

Q | obs.proposal.cycle

$S$ | obs.sequence

E | phot

E | phot.antennaTemp

Q | phot.calib

C | phot.color

Q | phot.color.excess

Q | phot.color.reddFree

E | phot.count

E | phot.fluence

E | phot.flux

Q|phot.flux.bol

E | phot.flux.density

E | phot.flux.density.sb

E | phot.flux.sb

E | phot.limbDark

E | phot.mag

$\mathrm{Q}$ | phot.mag.bc

Q | phot.mag.bol

Q | phot.mag.distMod

E | phot.mag.reddFree

E | phot.mag.sb

Q |phys

Q| phys.SFR

E | phys.absorption

Q| phys.absorption.coeff

Q | phys.absorption.gal

Q | phys.absorption.opticalDepth

Q | phys.abund

Q | phys.abund.Fe

Q | phys.abund.X

Q | phys.abund.Y

Q | phys.abund.Z

$\mathrm{Q}$ | phys.acceleration

Q | phys.albedo

Q | phys.angArea

Q | phys.angMomentum

$\mathrm{Q}$ | phys.angSize

Q | phys.angSize.smajAxis

Q | phys.angSize.sminAxis

$Q \mid$ phys.area

$S$ | phys.atmol

Q | phys.atmol.branchingRatio
| Atmosphere, atmospheric phenomena affecting an observation

| Atmospheric extinction

Atmospheric refraction angle

Calibration observation

| Related to flat-field calibration observation (dome, sky, ..)

Exposure

Region covered by the observation

I Image

Observer, discoverer

| Various observation or reduction parameter

| Observation proposal

| Proposal cycle

Sequence of observations, exposures or events

Photometry

Antenna temperature

Photometric calibration

| Color index or magnitude difference

Color excess

Dereddened, reddening-free color

Flux expressed in counts

Fluence

Photon flux

Bolometric flux

| Flux density (per wl/freq/energy interval)

Flux density surface brightness

Flux surface brightness

| Limb-darkening coefficients

Photometric magnitude

Bolometric correction

| Bolometric magnitude

| Distance modulus

Dereddened magnitude

Surface brightness in magnitude units

| Physical quantities

Star formation rate

Extinction or absorption along the line of sight

Absorption coefficient (e.g. in a spectral line)

Galactic extinction

Optical depth

Abundance

$\mathrm{Fe} / \mathrm{H}$ abundance

Hydrogen abundance

Helium abundance

| Metallicity abundance

Acceleration

Albedo or reflectance

Angular area

Angular momentum

| Angular size, width, diameter, dimension, extension, major minor axis, extraction radius

| Angular size, extent or extension of semi-major axis

Angular size, extent or extension of semi-minor axis

Area (in linear units)

Atomic and molecular physics

| Branching ratio 
Q | phys.atmol.collStrength

Q | phys.atmol.collisional

Q | phys.atmol.configuration

$\mathrm{Q}$ | phys.atmol.crossSection

Q | phys.atmol.element

Q | phys.atmol.excitation

$\mathrm{Q}$ | phys.atmol.final

Q | phys.atmol.initial

Q | phys.atmol.ionStage

$S$ | phys.atmol.ionization

Q | phys.atmol.lande

$S$ | phys.atmol.level

Q | phys.atmol.lifetime

Q | phys.atmol.lineShift

$\mathrm{Q}$ | phys.atmol.number

Q | phys.atmol.oscStrength

Q | phys.atmol.parity

$\mathrm{Q}$ | phys.atmol.qn

Q | phys.atmol.radiationType

Q | phys.atmol.symmetry

Q | phys.atmol.sWeight

Q | phys.atmol.sWeight.nuclear

$\mathrm{Q}$ | phys.atmol.term

Q | phys.atmol.transProb

$S$ | phys.atmol.transition

Q | phys.atmol.wOscStrength

Q | phys.atmol.weight

Q | phys.columnDensity

$S$ | phys.composition

$\mathrm{Q}$ | phys.composition.massLightRatio

Q | phys.composition.yield

$S$ | phys.cosmology

$\mathrm{Q}$ | phys.damping

Q | phys.density

$\mathrm{Q}$ | phys.dielectric

$\mathrm{Q}$ | phys.dispMeasure

$\mathrm{V}$ | phys.electField

$S$ | phys.electron

$\mathrm{Q}$ | phys.electron.degen

Q | phys.emissMeasure

Q | phys.emissivity

Q | phys.energy

Q | phys.energy.density

$\mathrm{Q}$ | phys.entropy

Q | phys.eos

$\mathrm{Q}$ | phys.excitParam

Q | phys.gauntFactor

Q | phys.gravity

Q | phys.ionizParam

Q | phys.ionizParam.coll

$\mathrm{Q}$ | phys.ionizParam.rad

E | phys.luminosity

Q | phys.luminosity.fun

$\mathrm{E} \mid$ phys.magAbs
| Collisional strength

Related to collisions

Configuration

Atomic / molecular cross-section

Element

Atomic molecular excitation parameter

Quantity refers to atomic/molecular final/ground state, level, ecc.

Quantity refers to atomic/molecular initial state, level, ecc.

Ion

| Related to ionization

Lande factor

Atomic level

| Lifetime of a level

Line shifting coefficient

Atomic number Z

Oscillator strength

| Parity

Atomic/molecular quantum number

| Type of radiation characterizing atomic lines (electric dipole/quadrupole, magnetic dipole)

| Type of nuclear spin symmetry

Statistical weight

Statistical weight for nuclear spin states

Atomic term

Atomic transition probability, Einstein A coefficient

Transition between states

| Weighted oscillator strength

Atomic weight

Column density

Quantities related to composition of objects

| Mass to light ratio

Mass yield

| Related to cosmology

Generic damping quantities

| Density (of mass, electron, ...)

Complex dielectric function

| Dispersion measure

Electric field

Electron

Electron degeneracy parameter

| Emission measure

Emissivity

Energy

| Energy-density

Entropy

Equation of state

| Excitation parameter U

| Gaunt factor/correction

Gravity

Ionization parameter

| Collisional ionization

Radiative ionization

| Luminosity

| Luminosity function

| Absolute magnitude 
Q | phys.magAbs.bol

$\mathrm{V}$ | phys.magField

$\mathrm{Q}$ | phys.mass

Q | phys.mass.loss

$\mathrm{Q}$ | phys.mol

Q | phys.mol.dipole

$\mathrm{Q}$ | phys.mol.dipole.electric

$\mathrm{Q}$ | phys.mol.dipole.magnetic

$\mathrm{Q}$ | phys.mol.dissociation

Q | phys.mol.formationHeat

Q | phys.mol.quadrupole

Q | phys.mol.quadrupole.electric

$S$ | phys.mol.rotation

$S$ | phys.mol.vibration

$S$ | phys.particle.neutrino

E | phys.polarization

Q | phys.polarization.circular

$\mathrm{Q}$ | phys.polarization.linear

Q | phys.polarization.rotMeasure

$\mathrm{Q}$ | phys.polarization.stokes

$\mathrm{Q}$ | phys.pressure

Q | phys.recombination.coeff

Q | phys.refractIndex

$Q$ | phys.size

Q | phys.size.axisRatio

Q | phys.size.diameter

$\mathrm{Q}$ | phys.size.radius

$\mathrm{Q}$ | phys.size.smajAxis

Q | phys.size.sminAxis

$\mathrm{Q}$ | phys.temperature

Q | phys.temperature.effective

Q | phys.temperature.electron

$\mathrm{Q}$ | phys.transmission

$\mathrm{V} \mid$ phys.veloc

$\mathrm{Q}$ | phys.veloc.ang

$\mathrm{Q}$ | phys.veloc.dispersion

$\mathrm{Q}$ | phys.veloc.escape

Q | phys.veloc.expansion

Q | phys.veloc.microTurb

Q | phys.veloc.orbital

Q | phys.veloc.pulsat

$\mathrm{Q}$ | phys.veloc.rotat

Q | phys.veloc.transverse

$Q$ | phys.virial

$\mathrm{Q} \mid$ pos

Q | pos.angDistance

Q | pos.angResolution

$\mathrm{Q}$ | pos.az

Q|pos.az.alt

$\mathrm{Q}$ |pos.az.azi

Q |pos.az.zd

$S$ | pos.barycenter

$S$ | pos.bodyrc

Q | pos.bodyrc.alt

Q | pos.bodyrc.lat

$\mathrm{Q}$ | pos.bodyrc.long
| Bolometric absolute magnitude

| Magnetic field

| Mass

| Mass loss

| Molecular data

| Molecular dipole

| Molecular electric dipole moment

Molecular magnetic dipole moment

| Molecular dissociation

Formation heat for molecules

| Molecular quadrupole

| Molecular electric quadrupole moment

Molecular rotation

Molecular vibration

| Related to neutrino

| Polarization degree (or percentage)

| Circular polarization

| Linear polarization

Rotation measure polarization

Stokes polarization

| Pressure

Recombination coefficient

Refraction index

| Linear size, length (not angular)

| Axis ratio (a/b) or (b/a)

| Linear diameter

Linear radius

| Linear semi major axis

Linear semi minor axis

| Temperature

| Effective temperature

Electron temperature

| Transmission (of filter, instrument, ...)

Space velocity

Angular velocity

| Velocity dispersion

Escape velocity

| Expansion velocity

| Microturbulence velocity

Orbital velocity

| Pulsational velocity

| Rotational velocity

| Transverse / tangential velocity

| Related to virial quantities (mass, radius, ..)

Position and coordinates

Angular distance, elongation

Angular resolution

| Position in alt-azimutal frame

| Alt-azimutal altitude

Alt-azimutal azimut

| Alt-azimutal zenith distance

Barycenter

Body related coordinates

Body related coordinate (altitude on the body)

Body related coordinate (latitude on the body)

| Body related coordinate (longitude on the body) 
S | pos.cartesian

$\mathrm{Q} \mid$ pos.cartesian.x

$Q \mid$ pos.cartesian.y

Q | pos.cartesian.z

$\mathrm{S}$ | pos.cmb

Q | pos.dirCos

$\mathrm{V}$ | pos.distance

$S \mid$ pos.earth

$\mathrm{Q}$ | pos.earth.altitude

$\mathrm{Q}$ | pos.earth.lat

$\mathrm{Q}$ | pos.earth.lon

S | pos.ecliptic

Q | pos.ecliptic.lat

Q | pos.ecliptic.lon

S | pos.eop

$\mathrm{Q}$ | pos.eop.nutation

Q |pos.ephem

$S \mid$ pos.eq

$\mathrm{Q}$ | pos.eq.dec

$\mathrm{Q}$ | pos.eq.ha

$\mathrm{Q}$ | pos.eq.ra

$\mathrm{Q}$ | pos.eq.spd

S | pos.errorEllipse

Q | pos.frame

S | pos.galactic

Q | pos.galactic.lat

Q | pos.galactic.lon

S | pos.galactocentric

$S$ | pos.geocentric

Q | pos.healpix

S | pos.heliocentric

$\mathrm{Q}$ | pos.HTM

$S$ | pos.lambert

S | pos.lg

S | pos.Isr

Q | pos.lunar

$Q$ | pos.lunar.occult

Q | pos.parallax

$\mathrm{Q}$ | pos.parallax.dyn

$\mathrm{Q}$ | pos.parallax.phot

Q | pos.parallax.spect

Q | pos.parallax.trig

$\mathrm{Q}$ | pos.phaseAng

V|pos.pm

$\mathrm{Q}$ | pos.posAng

$\mathrm{V}$ | pos.precess

S | pos.supergalactic

$\mathrm{Q}$ | pos.supergalactic.lat

$Q$ | pos.supergalactic.lon

$\mathrm{P} \mid$ pos.wcs

$P$ | pos.wcs.cdmatrix

$P \mid$ pos.wcs.crpix

$P$ | pos.wcs.crval

$P$ | pos.wcs.ctype

$P \mid$ pos.wcs.naxes
| Cartesian (rectangular) coordinates

| Cartesian coordinate along the $\mathrm{x}$-axis

Cartesian coordinate along the $y$-axis

| Cartesian coordinate along the z-axis

Cosmic Microwave Background reference frame

| Direction cosine

Linear distance

Coordinates related to Earth

Altitude, height on Earth above sea level

| Latitude on Earth

| Longitude on Earth

| Ecliptic coordinates

| Ecliptic latitude

| Ecliptic longitude

| Earth orientation parameters

| Earth nutation

Ephemeris

| Equatorial coordinates

| Declination in equatorial coordinates

| Hour-angle

| Right ascension in equatorial coordinates

| South polar distance in equatorial coordinates

Positional error ellipse

| Reference frame used for positions (FK5, ICRS,..)

Galactic coordinates

| Latitude in galactic coordinates

Longitude in galactic coordinates

| Galactocentric coordinate system

| Geocentric coordinate system

| Hierarchical Equal Area IsoLatitude Pixelization

| Heliocentric position coordinate (solar system bodies)

| Hierarchical Triangular Mesh

| Lambert projection

| Local Group reference frame

| Local Standard of Rest reference frame

| Lunar coordinates

Occultation by lunar limb

| Parallax

| Dynamical parallax

| Photometric parallax

Spectroscopic parallax

| Trigonometric parallax

| Phase angle, e.g. elongation of earth from sun as seen from a third cel. object

| Proper motion

| Position angle of a given vector

Precession (in equatorial coordinates)

| Supergalactic coordinates

| Latitude in supergalactic coordinates

| Longitude in supergalactic coordinates

| WCS keywords

| WCS CDMATRIX

WCS CRPIX

WCS CRVAL

WCS CTYPE

I WCS NAXES 
$\mathrm{P} \mid$ pos.wcs.naxis

$P$ | pos.wcs.scale

$Q$ | spect

Q | spect.binSize

S | spect.continuum

Q | spect.dopplerParam

E | spect.dopplerVeloc

E | spect.dopplerVeloc.opt

E | spect.dopplerVeloc.radio

E | spect.index

$\mathrm{S}$ | spect.line

E | spect.line.asymmetry

E | spect.line.broad

Q | spect.line.broad.Stark

E | spect.line.broad.Zeeman

$\mathrm{E}$ | spect.line.eqWidth

E | spect.line.intensity

E | spect.line.profile

Q | spect.line.strength

E | spect.line.width

$Q$ | spect.resolution

$S$ | src

$S$ | src.calib

S | src.calib.guideStar

$Q$ | src.class

$\mathrm{Q}$ | src.class.color

Q | src.class.distance

$Q$ | src.class.luminosity

$Q$ | src.class.richness

Q | src.class.starGalaxy

$Q$ | src.class.struct

Q | src.density

Q | src.ellipticity

Q | src.impactParam

$Q$ | src.morph

Q | src.morph.param

$\mathrm{Q}$ | src.morph.scLength

Q | src.morph.type

S | src.net

Q | src.orbital

Q | src.orbital.eccentricity

Q | src.orbital.inclination

Q | src.orbital.meanAnomaly

$\mathrm{Q}$ | src.orbital.meanMotion

Q | src.orbital.node

$Q$ | src.orbital.periastron

$Q \mid$ src.redshift

Q | src.redshift.phot

$Q$ | src.sample

Q | src.spType

$\mathrm{Q}$ | src.var

E | src.var.amplitude

$Q$ | src.var.index
I WCS NAXIS

WCS scale or scale of an image

Spectroscopy

Spectral bin size

Continuum spectrum

| Doppler parameter b

Radial velocity, derived from the shift of some spectral feature

| Radial velocity derived from a wavelength shift using the optical convention

| Radial velocity derived from a frequency shift using the radio convention

| Spectral index

Spectral line

Line asymmetry

Spectral line broadening

Stark line broadening coefficient

Zeeman broadening

| Line equivalent width

| Line intensity

Line profile

Spectral line strength S

Spectral line fwhm

Spectral (or velocity) resolution

Observed source viewed on the sky

| Calibration source

| Guide star

Source classification (star, galaxy, cluster...)

| Color classification

| Distance class e.g. Abell

Luminosity class

Richness class e.g. Abell

Star/galaxy discriminator, stellarity index

Structure classification e.g. Bautz-Morgan

Density of sources

Source ellipticity

| Impact parameter

Morphology structure

| Morphological parameter

Scale length for a galactic component (disc or bulge)

Hubble morphological type (galaxies)

Q Qualifier indicating that a quantity (e.g. flux) is background subtracted rather than total

| Orbital parameters

Orbit eccentricity

Orbit inclination

| Orbit mean anomaly

Mean motion

Ascending node

Periastron

Redshift

| Photometric redshift

Sample

Spectral type MK

Variability of source

Amplitude of variation

Variability index 
$Q$ | src.var.pulse

Q | stat

Q | stat.Fourier

Q | stat.Fourier.amplitude

$\mathrm{P}$ | stat.correlation

$\mathrm{P}$ | stat.covariance

$\mathrm{P}$ | stat.error

$\mathrm{P} \mid$ stat.error.sys

$\mathrm{Q}$ | stat.filling

Q | stat.fit

$\mathrm{P}$ | stat.fit.chi2

$P$ | stat.fit.dof

$\mathrm{P}$ | stat.fit.goodness

$S$ | stat.fit.omc

Q | stat.fit.param

$\mathrm{P}$ | stat.fit.residual

P | stat.likelihood

$S$ | stat.max

$\mathrm{S}$ | stat.mean

S | stat.median

$\mathrm{S}$ | stat.min

Q | stat.param

$\mathrm{Q}$ | stat.probability

$\mathrm{P}$ | stat.snr

$\mathrm{P}$ | stat.stdev

$S$ | stat.uncalib

$\mathrm{Q}$ | stat.value

$\mathrm{P}$ | stat.variance

$\mathrm{P}$ | stat.weight

Q | time

$\mathrm{Q}$ | time.age

Q | time.creation

Q | time.crossing

$\mathrm{Q} \mid$ time.duration

$\mathrm{Q} \mid$ time.end

$\mathrm{Q} \mid$ time.epoch

$\mathrm{Q}$ | time.equinox

Q | time.interval

Q | time.lifetime

Q | time.period

$\mathrm{Q}$ | time.phase

Q | time.processing

Q | time.publiYear

$Q$ | time.relax

$\mathrm{Q}$ | time.release

Q | time.resolution

$\mathrm{Q}$ | time.scale

Q | time.start
| Pulse

| Statistical parameters

| Fourier coefficient

Amplitude Fourier coefficient

Correlation between two parameters

Covariance between two parameters

Statistical error

Systematic error

| Filling factor (volume, time, ..)

Fit

| Chi2

Degrees of freedom

Goodness or significance of fit

Observed minus computed

Parameter of fit

Residual fit

| Likelihood

| Maximum or upper limit

| Mean, average value

Median value

| Minimum or lowest limit

| Generic statistical arameter

Probability

Signal to noise ratio

| Standard deviation

| Qualifier of a generic incalibrated quantity

Miscellaneous statistical value

Variance

Statistical weight

Time, generic quantity in units of time or date

Age

| Creation time/date (of dataset, file, catalogue,...)

Crossing time

Interval of time describing the duration of a generic event or phenomenon

End time/date of a generic event

Instant of time related to a generic event (epoch, date, Julian date, time stamp/tag,...)

| Equinox

Time interval, time-bin, time elapsed between two events, not the duration of an event

| Lifetime

Period, interval of time between the recurrence of phases in a periodic phenomenon

| Phase, position within a period

A time/date associated with the processing of data

Publication year

Relaxation time

The time/date data is available to the public

Time resolution

| Timescale

| Start time/date of generic event 


\section{Appendix B: Changes from previous versions}

\section{Changes from v1.22}

Text of par. 1.1 (2), last three lines;

List of em bands reordered according to $\mathrm{wl} /$ freq.

\section{Amendments/clarifications:}

Description changed in words:

phys.atmol.qn

\section{Additions:}

em.line.Hdelta, em.line.Lyalpha, em.line.CO

\section{Deletions/replacements:}

deleted: phys.mol.qn replaced by: phys.atmol.qn

\section{Changes from v1.21}

\section{Amendments/clarifications:}

Syntax flag changed in words:

phys.polarization

Description changed in words:

em.IR.FIR, em.IR.MIR, em.IR.NIR, em.line.OIII

\section{Changes from v1.2}

\section{Additions:}

spect.continuum

Changes from v1.11 (Rec20051231)

\section{Amendments/clarifications:}

Spelling: phys.atmol.sWeight

Syntax flag changed in words:

phys.atmol, spect.line

Description changed in words:

meta.dataset, obs.atmos, phot.color.reddFree, phys.size, phys.size.diameter, phys.size.radius, stat.param, stat.value, time, time.epoch, time.interval, time.period, time.phase

\section{Additions:}


em.bin, em.binSize, em.IR.FIR, em.IR.MIR, em.IR.NIR, em.UV.FUV, meta.abstract, meta.code.status, meta.email, meta.id.PI, meta.id.Col, meta.ref.ivorn, meta.ref.uri, obs.calib.flat, obs.exposure, obs.proposal, obs.proposal.cycle, obs.sequence, phys.atmol.symmetry, phys.atmol.sWeight.nuclear, phys.cosmology, phys.damping, phys.entropy, phys.particle.neutrino, phys.virial, spect.line.strength, src.calib, src.calib.guideStar, src.net, stat.filling, stat.probability, stat.uncalib, time.creation, time.duration, time.end, time.processing, time.publiYear, time.release, time.start

\section{Deletions/replacements:}

\begin{tabular}{|c|c|c|}
\hline phys.atmol.damping & phys.damping & $\begin{array}{c}\text { Atomic damping quantities } \\
\text { (van der Waals) }\end{array}$ \\
\hline phys.atmol.qn.I & phys.atmol.qn & $\begin{array}{l}\text { Nuclear spin quantum } \\
\text { number }\end{array}$ \\
\hline time.event.end & time.end & $\begin{array}{l}\text { End time of event or } \\
\text { phenomenon }\end{array}$ \\
\hline time.event.start & time.start & $\begin{array}{l}\text { Start time of event or } \\
\text { phenomenon }\end{array}$ \\
\hline time.expo.start & time.start;obs.exposure & Start time of exposure \\
\hline time.obs & time.duration; obs & $\begin{array}{l}\text { Observation on-time, } \\
\text { duration }\end{array}$ \\
\hline time.obs.end & time.end; obs & End time of observation \\
\hline time.obs.start & time.start;obs & Start time of observation \\
\hline
\end{tabular}

\section{Changes from v1.10}

1. A few minor changes to the text have been done

2. All UCD words are now compliant with the UCD recommendation. The corresponding changes are described below

3. The following words have been deprecated:

$\begin{array}{ll}\begin{array}{ll}\text { Deprecated UCD } \\ \text { phot.fluxDens }\end{array} & \begin{array}{l}\text { New corresponding UCD } \\ \text { phot.flux.density }\end{array} \\ \text { phot.fluxDens.sb } & \text { phot.flux.density.sb } \\ \text { phys.at* } & \text { phys.atmo|* } \\ \text { phys.atmol.coll } & \text { phys.atmol.collisional } \\ \text { phys.atmol.ion } & \text { phys.atmol.ionStage } \\ \text { phys.atmol.trans } & \text { phys.atmol.transition } \\ \text { phys.energyDensity } & \text { phys.energy.density } \\ \text { phys.massToLight } & \text { phys.composition.massLightRatio } \\ \text { phys.massYield } & \text { phys.composition.yield } \\ \text { spect.doppler } & \text { spect.dopplerParam }\end{array}$

4. The following word has been created: phys.composition 
5. The section Changes from previous versions has been reformatted

\section{Changes from v1.02}

1. Descriptions have been changed for the following words: em.line, instr.pixel, phys.gravity, pos.earth.altitude

2. The syntax flags changed for words: instr.filter, phys.angSize

3. The following words have been deprecated:

$\begin{array}{ll}\text { Deprecated UCD New corresponding UCD } \\ \text { instr.filter.transm } & \text { phys.transm;instr.filter } \\ \text { phys.mass.light } & \text { phys.massToLight } \\ \text { pos.resolution } & \text { pos.angResolution } \\ \text { pos.satellite } & \text { pos.bodyrc }\end{array}$

4. The following words have been created: phys.polarization.circular, phys.polarization.linear, phys.size.axisRatio, pos.bodyrc.alt, pos.bodyrc.lat, pos.bodyrc.long, time.event, time.event.end, time.event.start

\section{Changes from v1.01}

1. The following words have been restored to their previous spelling (v1.00): phot.fluDensity, phys.energDensity, phys.mYield, phot.fluxDensity, phys.energyDensity, phys.massYield

A note has been added to indicate that these words do not strictly comply with the UCD1+ Rec.

\section{Changes from v1.00}

1. Descriptions have been changed for the following words: em.IR.H, em.IR.J, em.IR.K, em.Xray.hard, em.X-ray.medium, em.X-ray.soft, em.gamma.hard, em.gamma.soft, em.opt.B, em.opt.I, em.opt.R, em.opt.U, em.opt.V, instr.bandpass, phot.count, phys.density, phys.mol.dipole.electric, phys.mol.dipole.magnetic, phys.mol.quadrupole.electric, pos.angDistance, pos.precess, src, src.class.distance, src.class.richness, src.class.starGalaxy, src.class.struct, time.expo, time.expo.end, time.expo.start, time.interval

2. The following words have been deprecated:

\author{
Deprecated UCD \\ instr.angRes \\ instr.obsty.site \\ instr.obsty.site.seeing \\ instr.spect \\ instr.spect.dispersion \\ instr.spect.order \\ instr.spect.resolution \\ instr.tel.focus \\ meta.fits.software \\ obs.air
}

\author{
New corresponding UCD \\ pos.resolution \\ pos.earth.altitude;instr.obsty \\ instr.obsty.seeing \\ instr \\ instr.dispersion \\ instr.order \\ spect.resolution \\ instr.tel.focalLength \\ meta.software \\ obs.atmos
}




\begin{tabular}{|c|c|}
\hline obs.air.extinction & obs.atmos.extinction \\
\hline obs.air.mass & obs.airMass \\
\hline phot.fluxDens & phot.fluDens \\
\hline hot.fluxDens.sb & phot.fluDens.sb \\
\hline phot.sb & phot.mag.sb \\
\hline phys.at.branchingRatio & phys.atmol.branchingRatio \\
\hline phys.at.crossSection & phys.atmol.crossSection \\
\hline phys.at.lineShift & phys.atmol.lineShift \\
\hline hys.at.moment & \\
\hline phys.at.moment.electric & phys.at.radiationType \\
\hline phys.at.moment.magnetic & phys.at.radiationType \\
\hline phys.at.qn.S & phys.at.qn \\
\hline phys.at.qn.L & phys.at.qn \\
\hline phys.at.qn.J & phys.at.qn \\
\hline phys.at.qn.F & phys.at.qn \\
\hline phys.atmol.state.final & phys.atmol.final \\
\hline phys.atmol.state.initial & phys.atmol.initial \\
\hline phys.massYield & phys.mYield \\
\hline phys.mol.quadrupole.magnetic & phys.at.radiationType \\
\hline phys.refraction & phys.refractlndex \\
\hline pos.az.ha & pos.eq.ha \\
\hline pos.earth.nutation & pos.eop.nutation \\
\hline spect.veloc & spect.dopplerVeloc \\
\hline src.fwhm & phys.angSize;src \\
\hline src.orbital.veloc & phys.veloc.orbital \\
\hline src.veloc & phys.veloc \\
\hline src.veloc.ang & phys.veloc.ang \\
\hline src.veloc.cmb & phys.veloc;pos.cmb \\
\hline src.veloc.dispersion & phys.veloc.dispersion \\
\hline src.veloc.escape & phys.veloc.escape \\
\hline src.veloc.expansion & phys.veloc.expansion \\
\hline src.veloc.lg & phys.veloc;pos.lg \\
\hline src.veloc.lsr & phys.veloc;pos.Isr \\
\hline src.veloc.microTurb & phys.veloc.microTurb \\
\hline src.veloc.pulsat & phys.veloc.pulsat \\
\hline src.veloc.rotat & phys.veloc.rotat \\
\hline
\end{tabular}

3. The syntax flags changed for words: instr.fov, instr.obsty, meta.file, phys.angSize, pos.cartesian, stat.fit.omc

4. The following words have been created: instr.dispersion, instr.order, instr.tel.focalLength, meta.curation, meta.software, meta.version, obs.atmos, obs.atmos.extinction, obs.airMass, obs.atmos.refractAngle, obs.calib, phys.at.radiationType, phys.atmol.branchingRatio, phys.atmol.crossSection, phys.atmol.lifetime, phys.atmol.lineShift, phys.energDensity, phys.refractIndex, phys.transmission, pos.eq.ha, pos.az.azi, pos.bodyrc, pos.cmb, pos.earth.altitude, pos.eop, pos.eop.nutation, pos.lg, pos.Isr, pos.phaseAng, pos.resolution, spect.resolution, spect.dopplerVeloc, spect.dopplerVeloc.radio, spect.dopplerVeloc.opt, 
src.orbital.meanMotion, phys.veloc, phys.veloc.ang, phys.veloc.dispersion, phys.veloc.escape, phys.veloc.expansion, phys.veloc.microTurb, phys.veloc.orbital, phys.veloc.pulsat, phys.veloc.rotat, phys.veloc.transverse, time.obs, time.obs.end, time.obs.start

\section{Changes from v0.2}

1. Section 1.2 has been simplified

2. 3 new syntax codes (E, C, V) have been introduced, and described in appendix A

3. The following words have been renamed :

$\begin{array}{ll}\text { Deprecated UCD } & \text { New corresponding UCD } \\ \text { em.line.21cm } & \text { em.line.HI } \\ \text { instr.ang-res } & \text { instr.angRes } \\ \text { instr.sky-level } & \text { instr.skyLevel } \\ \text { instr.sky-temp } & \text { instr.skyTemp } \\ \text { instr.antenna-temp } & \text { phot.antennaTemp } \\ \text { phys.absorption.gf } & \text { phys.gauntFactor } \\ \text { phys.at.einstein } & \text { phys.at.transProb } \\ \text { phys.at.level } & \text { phys.atmol.level } \\ \text { phys.dispMeas } & \text { phys.dispMeasure } \\ \text { phys.distance } & \text { pos.distance } \\ \text { phys.polarization.rotMeas phys.polarization.rotMeasure } \\ \text { phys.size.area } & \text { phys.area } \\ \text { pos.ang.separation } & \text { pos.angDistance } \\ \text { pos.ec } & \text { pos.ecliptic } \\ \text { pos.ec.lat } & \text { pos.ecliptic.lat } \\ \text { pos.ec.lon } & \text { pos.ecliptic.lon } \\ \text { pos.ee } & \text { pos.errorEllipse } \\ \text { pos.gal } & \text { pos.galactic } \\ \text { pos.gal.lat } & \text { pos.galactic.lat } \\ \text { pos.gal.lon } & \text { pos.galactic.lon } \\ \text { pos.sg } & \text { pos.supergalactic } \\ \text { pos.sg.lat } & \text { pos.supergalactic.lat } \\ \text { pos.sg.lon } & \text { pos.supergalactic.lon } \\ \text { src.class.star-galaxy } & \text { src.class.starGalaxy } \\ & \end{array}$

4. The following words have been created: instr.beam, meta.code.error, meta.id.part, phot.flux.sb, phys.angArea, phys.angSize, phys.angSize.smajAxis, phys.angSize.sminAxis, phys.area, phys.at.damping, phys.at.weight, phys.atmol.excitation, phys.mol.dissociation, phys.recombination.coeff, phys.size.smajAxis, phys.size.sminAxis, pos.cartesian, pos.cartesian.x, pos.cartesian.y, pos.cartesian.z, pos.distance, pos.eq.spd, pos.galactocentric, pos.geocentric, pos.healpix, pos.heliocentric, pos.HTM, pos.lambert, pos.satellite, spect.line.broad.Stark, spect.veloc, src.redshift.phot, stat.correlation, time.lifetime

5. Some words have been removed. The following table summarizes, when relevant, the suggested replacement to be used. 


\begin{tabular}{|c|c|}
\hline Deprecated UCD & New corresponding UCD \\
\hline instr.area & phys.area;instr \\
\hline instr.beam-width & phys.angSize;instr.beam \\
\hline meta.table.axis & phys.size;meta.table \\
\hline phot.color.Cous & phot.color \\
\hline phot.color.Gen & phot.color \\
\hline phot.color.Gunn & phot.color \\
\hline phot.color.JHN & phot.color \\
\hline phot.color.STR & phot.color \\
\hline phot.color.STR.c1 & phot.color \\
\hline phot.color.STR.b-y & phot.color \\
\hline phot.color.STR.m1 & phot.color \\
\hline phys.at.lineBroad & spect.line.broad \\
\hline phys.distance.compon & pos.distance;pos.cartesian.x (or y, z) \\
\hline phys.distance.gc & pos.distance;pos.galactocentric \\
\hline phys.electron.energy & phys.energy;phys.electron \\
\hline phys.extension & phys.angSize or phys.size \\
\hline phys.mass.fraction & phys.mass;arith.ratio \\
\hline phys.polarization.posAng & pos.posAng;phys.polarization \\
\hline pos.ang & \\
\hline pos.det & pos.cartesian;instr.det \\
\hline pos.eq.dec.arcsec & \\
\hline pos.eq.ra.minutes & \\
\hline pos.eq.ra.seconds & \\
\hline pos.gal.compon & pos.cartesian;pos.galactic \\
\hline pos.pm.dec & pos.pm;pos.eq.dec \\
\hline pos.pm.ra & pos.pm;pos.eq.ra \\
\hline pos.precess.dec & pos.precess;pos.eq.dec \\
\hline pos.precess.ra & pos.precess;pos.eq.ra \\
\hline pos.proj & \\
\hline pos.sg.compon & pos.cartesian;pos.supergalactic \\
\hline src.orbital.energy & phys.energy;src.orbital \\
\hline src.orbital.separation & pos.angDistance;src.orbital \\
\hline src.orbital.size & phys.size;src.orbital \\
\hline src.separation & pos.angDistance;src \\
\hline src.veloc.compon & src.veloc;pos.cartesian \\
\hline src.veloc.gc & src.veloc;pos.galactocentric \\
\hline src.veloc.geoc & src.veloc;pos.geocentric \\
\hline src.veloc.hc & src.veloc;pos.heliocentric \\
\hline
\end{tabular}

\section{Changes from v0.1}

1. Descriptions of the words were improved.

2. Designation of commonly used lines have been moved to em.line. *. As a consequence, terms like em.IR.K.Brgamma or spect.index.Hbeta have been removed. 
3. phys.at and phys.mol have been completely reorganized to improve the overall description of this domain. A new branch phys.atmol has been introduced to group concepts shared between phys.at and phys.mol.

4. The phot.color section was significantly simplified.

5. Missing nodes of the tree were added (e.g. em.gamma, em.mm, pos.sg).

6. Creation of new words: em.wavenumber, meta.ucd, stat.error.sys

7. Typos were corrected in em.opt.. ${ }^{*}$ units and a few other descriptions.

\section{References}

[1] R. Hanisch, Resource Metadata for the Virtual Observatory, http://www.ivoa.net/Documents/latest/RM.html

[2] R. Hanisch, M. Dolensky, M. Leoni, Document Standards Management: Guidelines and Procedure, http://www.ivoa.net/Documents/latest/DocStdProc.html 